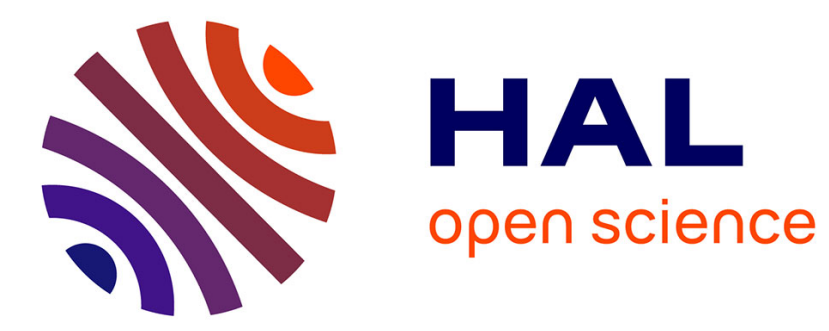

\title{
Uncertainty in historical Value-at-Risk: an alternative quantile-based risk measure
}

\author{
Dominique Guegan, Bertrand K. Hassani, Kehan Li
}

\section{To cite this version:}

Dominique Guegan, Bertrand K. Hassani, Kehan Li. Uncertainty in historical Value-at-Risk: an alternative quantile-based risk measure. 2016. halshs-01277880

\section{HAL Id: halshs-01277880 \\ https://shs.hal.science/halshs-01277880}

Submitted on 23 Feb 2016

HAL is a multi-disciplinary open access archive for the deposit and dissemination of scientific research documents, whether they are published or not. The documents may come from teaching and research institutions in France or abroad, or from public or private research centers.
L'archive ouverte pluridisciplinaire HAL, est destinée au dépôt et à la diffusion de documents scientifiques de niveau recherche, publiés ou non, émanant des établissements d'enseignement et de recherche français ou étrangers, des laboratoires publics ou privés. 


\section{Documents de Travail du \\ PANTHÉON SORBONNE Centre d'Economie de la Sorbonne}

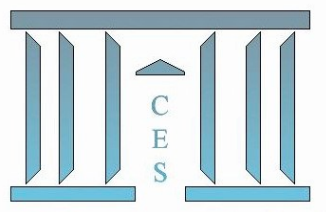

\section{Uncertainty in historical Value-at-Risk: an alternative quantile-based risk measure}

Dominique GuEGAN, Bertrand HAssAnI, Kehan LI

2016.06

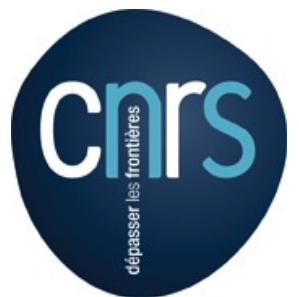




\title{
Uncertainty in historical Value-at-Risk: an alternative quantile-based risk measure
}

\author{
Dominique Guégan ${ }^{\mathrm{a}}$, Bertrand Hassani ${ }^{\mathrm{b}}$, Kehan $\mathrm{Li}^{\mathrm{a}, \mathrm{c}, *}$ \\ ${ }^{a}$ Université Paris 1 Panthéon-Sorbonne, CES UMR 8174. 106 bd l'Hopital 75013, Paris, \\ France. \\ ${ }^{b}$ Grupo Santander and Université Paris 1 Panthéon-Sorbonne, CES UMR 8174. \\ ${ }^{c}$ This work was achieved through the Laboratory of Excellence on Financial Regulation \\ (Labex ReFi) supported by PRES heSam under the reference ANR10LABX0095. It \\ benefited from a French government support managed by the National Research Agency \\ (ANR) within the project Investissements d'Avenir Paris Nouveaux Mondes (investments \\ for the future Paris New Worlds) under the reference ANR11IDEX000602.
}

\begin{abstract}
The financial industry has extensively used quantile-based risk measures relying on the Value-at-Risk $(V a R)$. They need to be estimated from relevant historical data set. Consequently, they contain uncertainty. We propose an alternative quantile-based risk measure (the Spectral Stress $V a R$ ) to capture the uncertainty in the historical $V a R$ approach. This one provides flexibility to the risk manager to implement prudential regulatory framework. It can be a $V a R$ based stressed risk measure. In the end we propose a stress testing application for it.
\end{abstract}

Keywords: Historical method, Uncertainty, Value-at-Risk, Stress risk measure, Tail risk measure, Prudential financial regulation, Stress testing

JEL: G28, G32, C14

\section{Introduction}

The financial industry has extensively used quantile-based risk measures based on the Value-at-Risk $(V a R)$. In statistical terms, the $V a R$ is a quantile reserve,

\footnotetext{
${ }^{*}$ Corresponding author.

Email addresses: dominique.guegan@univ-paris1.fr (Dominique Guégan), bertrand.hassani@gmail.com (Bertrand Hassani), kehanleex@gmail.com (Kehan Li)

Preprint submitted to Finance Research Letters

January 14, 2016
} 
often using the $p^{t h}(p \in[0,1])$ percentile of the loss distribution. Typically the $5 \quad V a R$ is not known with certainty and needs to be estimated from sample estimators of relevant observations. Bignozzi and Tsanakas (2015) [6] point out that the observations are often very small creating statistical error, which means that the values of sample estimators can diverge substantially from the true values. Jorion (1996) [10] calls it the risk in Value-at-Risk itself. Pérignon and Smith

${ }_{10}$ (2010) 12 find that historical $V a R$ is the most popular $V a R$ method, as $73 \%$ of the banks report their $V a R$ estimation methodologies using historical $V a R$.

Our paper proposes an alternative risk measure based on the historical $V a R$. A confidence interval (CI) is considered to integrate the uncertainty contained 15 in the historical $V a R$. It is a tail risk measure at multiple confidence levels (Alexander, Baptista and Yan (2015) [2]). It provides the flexibility to the risk manager to implement a prudential regulatory framework (Basel Committee on Banking Supervision (BCBS) [3] and Acharya (2009) [1]). Additionally, it can be a $V a R$ based stressed risk measure based on a continuous 12-month period 20 of significant financial stress following the requirement of the Basel Committee (BCBS (2011) [5]). We propose a stress testing application for this risk measure.

Numerous papers discussed the confidence interval of the $V a R$. For example, Pritsker (1997) [13] computes a nonparametric CI to evaluate the accuracy of 25 different $V a R$ approaches. Christoffersen and Gonçalves (2005) [7] assess the precision of $V a R$ forecast by using bootstrap prediction intervals. Jorion (1996) [10] provides the asymptotic standard error and confidence bands for sample quantile, assuming the loss distribution is known. All these approaches mainly use their CI (provided by asymptotic result or bootstrap) as a complementary so tool to assess the quality of the $V a R$. In our work we consider another approach to build the CI (we do not assume that the loss distribution is known and we do not use simulation). We use an asymptotic result and a parametric approach. 
We focus on a fat-tail distribution 1 to capture historical stress information, in order to build a stressed risk measure. Finally we use the lower (or upper) bound of CI directly as one boundary of our risk measure.

This paper is organised as follows. Section 2 describes our risk measure. Section 3 proposes a stress testing application for the risk measure. Section 4 concludes.

${ }_{40}$ 2. The Spectral Stress $\operatorname{VaR}$ measure

Consider a financial variable $X$ (for example the return of a portfolio, the return of a risk factor or an operational loss). Assume that it is a r.v. with a cumulative distribution function (cdf) $F_{\boldsymbol{\theta}}\left(f_{\boldsymbol{\theta}}\right.$ is the associated probability density function (pdf) and $\boldsymbol{\theta}$ are the parameters). Let $X_{1}, \ldots, X_{n}$ be the historical information 45 set of $X$ with length $n$.

As in Christoffersen and Gonçalves (2005) [7, we define the historical VaR $\left(X_{([n p]+1)}\right)$ as the $(1-p)$ th empirical quantile of the losses data. We fit a panel of distributions using $X_{1}, \ldots, X_{n}$ to compute the estimators of $\boldsymbol{\theta}$, denoted

50 $\hat{\boldsymbol{\theta}}$. Then $F_{\hat{\boldsymbol{\theta}}}$ and $f_{\hat{\boldsymbol{\theta}}}$ are the estimators of $F_{\boldsymbol{\theta}}$ and $f_{\boldsymbol{\theta}}$. Given confidence levels $0<p<1$ and $0<q<12$, we build a confidence interval $C I_{p, q}$ around $X_{([n p]+1)}$ (Rao (2002) [14]; Guégan, Hassani and Li (2015) 8]):

$$
X_{([n p]+1)} \in\left[F_{\hat{\boldsymbol{\theta}}}^{-1}(p)-z_{\frac{1+q}{2}} \sqrt{\widehat{V}}, \quad F_{\hat{\boldsymbol{\theta}}}^{-1}(p)+z_{\frac{1+q}{2}} \sqrt{\widehat{V}}\right]
$$

where

$$
\widehat{V}=\frac{p(1-p)}{\left[f_{\hat{\boldsymbol{\theta}}}\left(F_{\hat{\boldsymbol{\theta}}}^{-1}(p)\right)\right]^{2} n} .
$$

\footnotetext{
${ }^{1} \mathrm{~A}$ fat-tailed distribution has the property that it exhibits large kurtosis or has power law decay in the tail of the distribution.

${ }^{2} p$ is the confidence level of historical $V a R$ and $q$ is the confidence level of its confidence interval.
} 
and $z_{\frac{1+q}{2}}$ is the $\frac{1+q}{2} t h$ quantile of standard Gaussian distribution. According to the expression (1), $C I_{p, q}$ depends on $n, \hat{f}, p$ and $q$.

In practice for a sequence $p_{1}<p_{2}<\ldots<p_{k}$, given $\left\{q_{i}\right\}_{i=1, \ldots, k}$, we compute the sequences $\left\{F_{\hat{\boldsymbol{\theta}}}^{-1}\left(p_{i}\right)\right\}$ and $C I_{p_{i}, q_{i}}$ for $i=1, \ldots, k$. We define an area delineated by $F_{\hat{\boldsymbol{\theta}}}^{-1}\left(p_{i}\right)$ and the lower (or upper) bound of $C I_{p_{i}, q_{i}}$ for $i=1, \ldots, k$. We call this area the Spectral Stress $\operatorname{VaR}$ measure (SSVaR). Figure 1 provides a graph of the SSVaR. The lower (green) and upper (red) curves correspond to the boundaries of $C I_{p_{i}, q_{i}}$ for $\left\{p_{i}\right\}$ and $\left\{q_{i}\right\}, i=1, \ldots, k$. The black curve in the middle is associated to the sequence of $\left\{\left\{F_{\hat{\boldsymbol{\theta}}}^{-1}\left(p_{i}\right)\right\}\right.$ for $i=1, \ldots, k$. The black shadow area is the SSVaR.

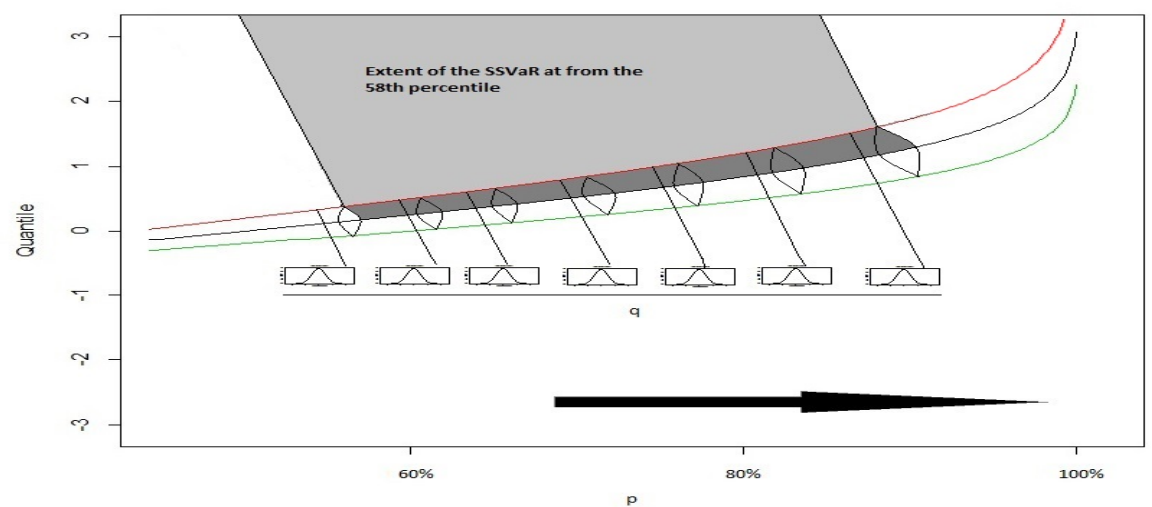

Figure 1: The lower (green) and upper (red) curves correspond to the boundaries of $C I_{p_{i}, q_{i}}$ for $\left\{p_{i}\right\}$ and $\left\{q_{i}\right\}, i=1, \ldots, k$. The black curve in the middle is associated to the sequence of $\left\{\left\{F_{\hat{\boldsymbol{\theta}}}^{-1}\left(p_{i}\right)\right\}\right.$ for $i=1, \ldots, k$. The black shadow area is the SSVaR. When the values of $q_{i}$ change, SSVaR can shift to the grey area.

It is important to point out that when the risk manager has to work within the prudential regulatory framework, he can choose higher $q_{i}$ leading to shift the SSVaR to the grey area. Also, he can shift the SSVaR to the grey area by choosing a fat-tail $\hat{f}$. In fact a fat-tail $\hat{f}$ can take more stress information from 
70 a period of significant financial turmoil than a thin tail fit. Consequently the $\mathrm{SSVaR}$ is a stressed risk measure in essence.

\section{A stress testing application of the SSVaR}

During recent crisis, some investors have suffered considerable losses due to ex-

75 treme events. Consequently there has been a growing literature on stress testing. Specially, banks that use the $V a R$ approach must have in place a rigorous stress testing program (BCBS (2005) [4]). In response, we propose a SSVaR measure applicable to the stress testing. The result of the stress testing is also a criteria to choose a reasonable $\hat{f}$ to build the SSVaR, which we can use first as an alert indicator.

To explain our purpose we consider a fictive financial institution. This one holds a Chinese market portfolio (that is, the same stock components and weights as the Shanghai Stock Exchange Composite Index (SHCOMP)). We compute the SSVaR using the daily return of SHCOMP from 29/06/2007 to 20/06/2008 (it contains 246 points and we call it $\Omega_{1}$ ). The historical $V a R$ of $\Omega_{1}$ are computed. For the stress testing, we compute the empirical quantiles on the daily return of SHCOMP from 01/12/2014 to 09/11/2015 (it contains 241 points and we call it $\Omega_{2}$ ). Table 1 provides the empirical statistics of the data sets. It shows these two data sets are left skewed and leptokurtic (Kurtosis > 3). The distributions which characterise these two data sets need to have these properties. In the following we build SSVaR using $\Omega_{1}$, with Gaussian distribution as a benchmark and Normal-inverse Gaussian distribution (NIG, Godin (2012) [9]).

95 To take into account the left tail market risk, we use $0.01 \leq p_{i} \leq 0.1$ and fixed $q=0.95$. We build the SSVaR for $\Omega_{1}$ using Gaussian distribution $3^{3}$ and NIG 4

\footnotetext{
${ }^{3}$ The mean equals to -0.0017 and variance equals to 0.0007 .

${ }^{4}$ The tail parameter parameter equals to 90.63 , skewness parameter equals to -25.73 ,
} 
Table 1: Empirical statistics of SHCOMP daily returns from 29/06/2007 to 20/06/2008 $\left(\Omega_{1}\right)$ and from $01 / 12 / 2014$ to $09 / 11 / 2015\left(\Omega_{2}\right)$

\begin{tabular}{lllll}
\hline & Mean & Variance & Skewness & Kurtosis \\
\hline$\Omega_{1}(n=246)$ & -0.0017 & 0.0007 & -0.3796 & 3.7876 \\
$\Omega_{2}(n=241)$ & 0.0010 & 0.0007 & -1.0509 & 5.0698 \\
\hline
\end{tabular}

In Figure 2, on the left graph the dashed (blue and green) lines are the upper and lower bounds of the SSVaR corresponding to the Gaussian distribution. On the right graph the dashed (blue and green) lines are the upper and lower bounds of the SSVaR corresponding to the NIG distribution. In these two graphs, the solid (red) lines are the historical $V a R$ and the solid-dot (brown) lines are the empirical quantiles for $\Omega_{2}$.

In Figure 2, the left graph suggests that the SSVaR based on a Gaussian distribution underestimates the risk computed using $\Omega_{1}$ and $\Omega_{2}$, because the left part of the historical $V a R$ and the empirical quantiles are outside the SSVaR. The right graph shows that the SSVaR built using a NIG distribution permits to control the risk more efficiently since they are almost inside the SSVaR. Additionally, ignoring the uncertainty in the historical $\operatorname{VaR}$ (that is, use the empirical quantiles directly as the risk measure) leads to underestimate the risk computed using $\Omega_{2}$, because the left part of the empirical quantiles is lower than the historical $\operatorname{VaR}$.

In practice, the SSVaR is a improvement risk measure of historical $V a R$. The risk manager can use it directly to allocate capital reserve to the risk of measurement uncertainty. Additionally, it can be a stressed and tail risk measure providing flexibility to the risk manager to work within the prudential regulatory framework.

location parameter equals to 0.0155 and scale parameter equals to 0.058 . 

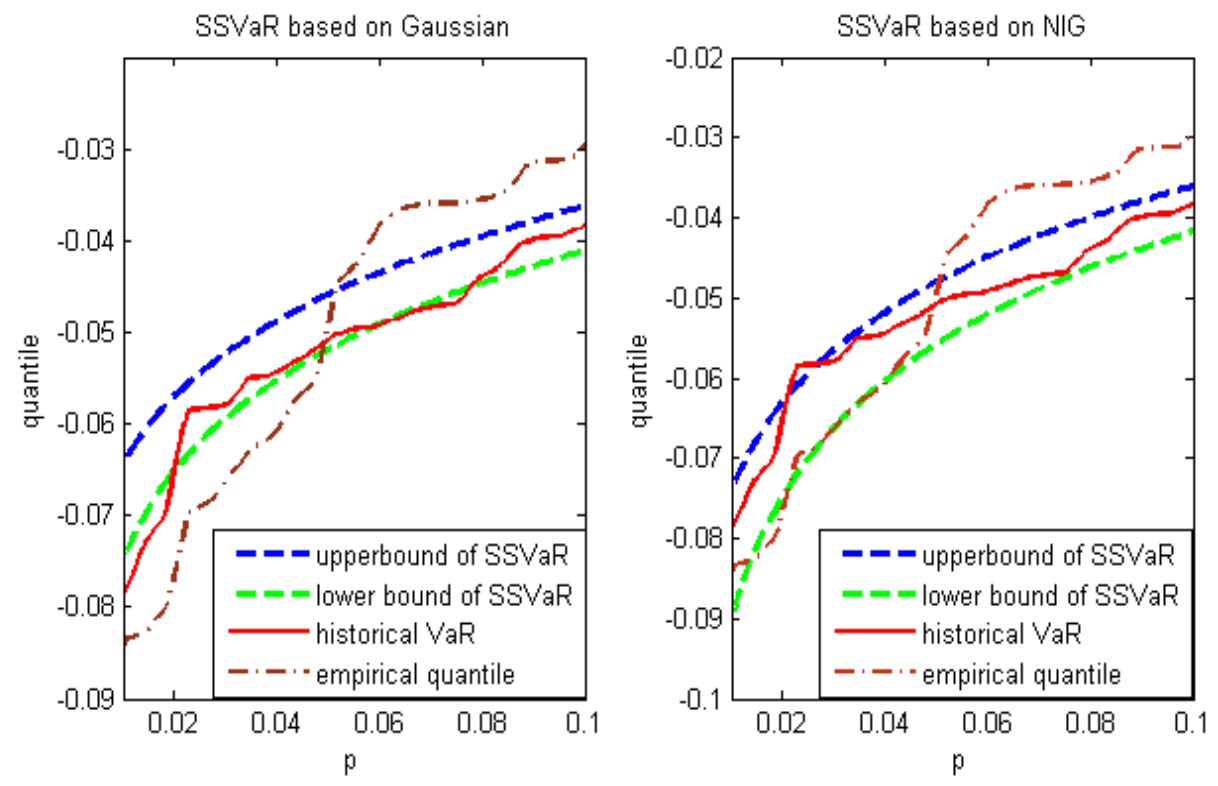

Figure 2: We use $0.01 \leq p_{i} \leq 0.1$ and fixed $q=0.95$ and build the SSVaR for $\Omega_{1}$ using Gaussian distribution (mean -0.0017 and variance 0.0007 ) and NIG (with tail parameter parameter equalling to 90.63 , skewness parameter equalling to -25.73 , location parameter equalling to 0.0155 and scale parameter equalling to 0.058). In Figure 2, on the left graph the dashed (blue and green) lines are the upper and lower bounds of the SSVaR corresponding to the Gaussian distribution. On the right graph the dashed (blue and green) lines are the upper and lower bounds of the SSVaR corresponding to the NIG distribution. In these two graphs, the solid (red) lines are the historical $V a R$ and the solid-dot (brown) lines are the empirical quantiles for $\Omega_{2}$.

\section{Conclusion}

In this article, we propose an alternative quantile-based risk measure SSVaR, to integrate the uncertainty from the historical $V a R$. Additionally, it is a tail risk measure. Also, it provides the flexibility to the risk manager to implement prudential regulatory framework. It can be a $V a R$ based stressed risk measure.

${ }_{125}$ Additionally, We propose a stress testing application for the SSVaR, by illustrating the magnitude of the exceptions based on the empirical quantile of two 
data sets from SHCOMP. The results suggest that ignoring the uncertainty in the historical $V a R$ leads to underestimate risks. Also, we observe that when the data sets are skewed and leptokurtic, risk manager needs to fit a skewed and leptokurtic distribution to build SSVaR. It leads to control the risk efficiently.

As the purpose of a forthcoming paper, some improvements of this approach could be done. Indeed, the expression (1) relies on the assumption of independence for $X_{1}, \ldots, X_{n}$ (Rao (2002) [14). Nevertheless, we can extend the results can be used directly for the operational risks which are mainly independent. For other risks we can calibrate dynamics on $X_{1}, \ldots, X_{n}$, like $X_{t}=f\left(X_{t-1}\right)+\epsilon_{t}$ where $\epsilon_{t}$ is a white noise. Then we build the SSVaR using the residuals $\left\{\epsilon_{t}\right\}$, and the time series modelling can be used to introduce dynamics inside the SSVaR.

\section{References}

[1] V. V. Acharya. A theory of systemic risk and design of prudential bank regulation. Journal of Financial Stability, 5(3):224-255, 2009.

[2] G. J. Alexander, A. M. Baptista, and S. Yan. On regulatory responses to the recent crisis: An assessment of the basel market risk framework and the volcker rule. Financial Markets, Institutions Instruments, 24(2-3):87-125, 2015.

[3] Basel Committee on Banking Supervision. A New Capital Adequacy Framework. Technical Report, 1999.

[4] Basel Committee on Banking Supervision. Amendment to the Capital Accord to incorporate market risks. Working Paper, 2005.

[5] Basel Committee on Banking Supervision. Basel III: A global regulatory framework for more resilient banks and banking systems. Working Paper, 2011. 
[6] V. Bignozzi and A. Tsanakas. Parameter uncertainty and residual estimation risk. Journal of Risk and Insurance, page Forthcoming. ， 2015.

[7] P. Christoffersen and S. Gonçalves. Estimation risk in financial risk management. Journal of Risk, 7(3):1-28, 2005.

[8] D. Guégan and B. Hassani and K. Li. The Spectral Stress VaR (SSVaR). Centre d'Économie de la Sorbonne Working Paper, 2015.

[9] F. Godin, S. Mayoral, and M. Morales. Contingent claim pricing using a normal inverse gaussian probability distortion operator. The Journal of Risk and Insurance, 79(3):841-866, 2012.

[10] P. Jorion. Risk2: Measuring the risk in value at risk. Financial Analysts Journal, 52(6):47-56, 1996.

[11] M. R. Leadbetter and G. Lindgren and H. Rootzén. Extremes and Related Properties of Random Sequences and Processes. Springer-Verlag, New York, 1983.

[12] C. Pérignon and D.R. Smith. The level and quality of value-at-risk disclosure by commercial banks. Journal of Banking \& Finance, 34(2):362-377, 2010.

[13] M. Pritsker. Evaluating value at risk methodologies: Accuracy versus computational time. Journal of Financial Services Research, 12(2-3):201-242, 1997.

[14] C. R. Rao. Linear statistical inference and its applications. John Wiley \& Sons, Hoboken, 2 edition, 2002. 